\title{
Different classes of small RNAs are essential for head regeneration in the planarian Dugesia japonica
}

\author{
Zhonghong $\mathrm{Cao}^{1 *+} \mathbb{D}$, David Rosenkranz ${ }^{2 \dagger}$, Suge $\mathrm{Wu}^{1 \dagger}$, Hongjin Liu', Qiuxiang Pang ${ }^{1}$, Xiufang Zhang ${ }^{1}$,

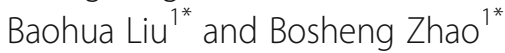

\begin{abstract}
Background: Planarians reliably regenerate all body parts after injury, including a fully functional head and central nervous system. But until now, the expression dynamics and functional role of miRNAs and other small RNAs during the process of head regeneration are not well understood. Furthermore, little is known about the evolutionary conservation of the relevant small RNAs pathways, rendering it difficult to assess whether insights from planarians will apply to other taxa.

Results: In this study, we applied high throughput sequencing to identify miRNAs, tRNA fragments and piRNAs that are dynamically expressed during head regeneration in Dugesia japonica. We further show that knockdown of selected small RNAs, including three novel Dugesia-specific miRNAs, during head regeneration induces severe defects including abnormally small-sized eyes, cyclopia and complete absence of eyes.

Conclusions: Our findings suggest that a complex pool of small RNAs takes part in the process of head regeneration in Dugesia japonica and provide novel insights into global small RNA expression profiles and expression changes in response to head amputation. Our study reveals the evolutionary conserved role of miR-124 and brings further promising candidate small RNAs into play that might unveil new avenues for inducing restorative programs in nonregenerative organisms via small RNA mimics based therapies.
\end{abstract}

Keywords: Dugesia japonica, Head regeneration, Micro RNAs, Piwi-interacting RNAs, tRNA fragments, miR-124

\section{Background}

The limited regenerative capabilities of most vertebrates including humans, particularly regarding damage to the central nervous system (CNS), call for effective therapies that foster the replacement or healing of wounded tissues. Therefore it is imperative to understand the molecular mechanisms of

\footnotetext{
* Correspondence: zhcao@sdut.edu.cn; ppliew@szu.edu.cn;

zhaobosheng@sdut.edu.cn

'Zhonghong Cao, David Rosenkranz and Suge Wu contributed equally to this work.

${ }^{1}$ School of Life Sciences, Shandong University of Technology, 266 Xincun Western Road, Zibo 255049, People's Republic of China

Full list of author information is available at the end of the article
}

regeneration and signal networks that induce and promote this complex process.

Planarian flatworms possess an extensive potential of regeneration and are one of the few animal species that can easily regenerate their head after decapitation including the complete neoformation of a functional brain within 7 days [1-4]. Despite their relatively simple morphology, planarians have a highly structured CNS featuring a true brain consisting of a large number of different neuronal cell types [5, 6], a well-defined adult stem cell population comprising roughly $30 \%$ of all CNS cells and a clear anterior-posterior $(\mathrm{A} / \mathrm{P})$ polarity that is maintained during regeneration [7-9]. Moreover, planarians share more

(c) The Author(s). 2020 Open Access This article is licensed under a Creative Commons Attribution 4.0 International License, which permits use, sharing, adaptation, distribution and reproduction in any medium or format, as long as you give appropriate credit to the original author(s) and the source, provide a link to the Creative Commons licence, and indicate if changes were made. The images or other third party material in this article are included in the article's Creative Commons licence, unless indicated otherwise in a credit line to the material. If material is not included in the article's Creative Commons licence and your intended use is not permitted by statutory regulation or exceeds the permitted use, you will need to obtain permission directly from the copyright holder. To view a copy of this licence, visit http://creativecommons.org/licenses/by/4.0/ The Creative Commons Public Domain Dedication waiver (http://creativecommons.org/publicdomain/zero/1.0/) applies to the data made available in this article, unless otherwise stated in a credit line to the data. 
genes with vertebrates than other popular model organisms such as Drosophila melanogaster or Caenorhabditis elegans do [10], and many genes expressed in the planarian CNS are highly conserved in humans [11].

Recent work has shown that a planarian reaches three main milestones to restore its head. First, it must determine that it is missing a head rather than a tail. Second, the anterior pole must be formed at the anterior tip. Third, the missing tissues must be reconstructed [12]. This process involves two systems, i) Pluripotent neoblasts that can generate new cell types and ii) muscle cells that provide positional instructions during the regeneration process. The subepidermal planarian muscle tissue is a major source of the positional information that orchestrates tissue turnover and regeneration programs $[13,14]$. During regeneration, wht1 is expressed in the posterior pole [14-16] and knockdown of Wnt signaling results in animals that regenerate heads at all blastemas, while animals with constitutively active Wnt signaling regenerate tails rather than heads [17-19], and the polarized activation of notum in muscle cells at anterior-facing wounds in turn steers Wnt function [15, 16]. In addition to Wnt signaling, the hedgehog ( $\mathrm{Hh})$ signaling pathway represents another essential regulator during head regeneration, and animals with defective $\mathrm{Hh}$ signaling show severe A/P patterning defects, completely fail to regenerate heads, or ectopically regenerate tails at anterior-facing wounds $[20,21]$. The anterior regeneration pole is formed by a cluster of collagen ${ }^{+}$cells which co-express notum, follistatin $\left(f_{s t}\right)$ genes and the transcription factors foxD and zicl, and knockdown of these anteriorly expressed genes results in impaired head regeneration, yet without induction of ectopic posterior markers at anterior-facing blastemas [22-24]. Finally, a number of other factors such as CHD4, p53, and MEX3, coe, lhx1/5-1, pitx, klf, and pax3/7 have been shown to be required for head regeneration and regeneration of multiple neuron subtypes [4, 25-27]. However, the factors that regulate the spatiotemporal expression of these genes, which are crucial for the proper patterning of the planarian head, are not known.

Micro RNAs (miRNAs) are small, non-coding RNAs that act in post-transcriptional gene regulation and play important roles in virtually all biological processes including stem cell self-renewal, proliferation and differentiation $[28,29]$ and a number of studies have shown that miRNAs are critical regulators of regeneration [30-33]. Contrasting their functional importance, our knowledge of miRNA expression patterns and function during head regeneration in planarians is far from being complete [34-36]. In addition, our current understanding is based on experiments in the planarian Schmidtea mediterranea (S. mediterranea), presuming but not having any evidence for an evolutionary conservation. However, finding evolutionary conserved mechanisms is vital when the long-term objective of research that uses animal model systems is to gain insights that in the end are applicable to humans. The planarian Dugesia japonica (D. japonica) possess equally impressive capacities to reliably regenerate a head including a functional brain within days, and, although the genus Dugesia represents the closest known relative to the genus Schmidtea, both taxons have evolved independently for at least the last 43 million years [37]. Hence, mechanisms that are not conserved across these two planarian species will, apart from being interesting in an evolutionary context, likely have no implications for human therapeutics.

In this study we monitor small RNA (sRNA) expression profiles during head regeneration in $D$. japonica applying state-of-the-art high throughput sequencing technologies. We identify homologous and Dugesia-specific miRNA genes and provide a detailed analysis of the major sRNA classes in $D$. japonica. We describe the dynamic sRNA expression patterns during head regeneration and compare the observed patterns with that of $S$. mediterranea with the aim of identifying conserved regulatory regimes. Finally, we validate the functional importance of selected upregulated sRNAs including miRNAs and tRNA derived fragments (tRFs) by demonstrating that their knockdown in head regenerating animals severely impairs regeneration, resulting in eye-less heads, cyclopia and other phenotypic defects.

\section{Results}

\section{Detection of 4 novel $D$. japonica miRNA genes}

For each library, 8.6 to 14.7 million reads were successfully mapped to the genome of D. japonica. Based on our small RNA transcriptome data we identified 36 miRNA genes with ShortStack [39], 32 of which have homologs in the planarian $S$. mediterranea while the remaining four miRNA genes lack sequence homology to other annotated miRNA genes in miRBase, thus representing either novel miRNA genes aquired on the lineage of D. japonica, or alternatively ancestral miRNA genes that were lost in $S$. mediterranea (dja-miR-novel$1 /-2 /-3 /-4$, Supplementary Table S1). We checked for a significant enrichment of specific GO terms assigned to the putative targets of the novel miRNAs and found that dja-miR-novel-1 targets $(n=205)$ are particularly enriched for genes involved in apoptosis and regulation of JNK cascade. Dja-mir-novel-2 targets $(n=136)$ are enriched for genes involved in membrane organization and dja-mir-novel-3 targets $(n=105)$ show an enrichment for genes connected to photoreception. For djamir-novel-4 we did not observe any enrichment, possibly due to the high number of predicted targets $(n=715$, Supplementary Table S2). 
miRNAs, tRFs and piRNAs represent the major sRNA fractions in $D$. japonica

Generally, the annotation of small RNAs using the unitas annotation pipeline [41] yielded similar fractions of small RNA classes across the different time points of sampling after head amputation (Fig. 1a and b, Supplementary Table S3). While $41-45 \%$ of the mapped reads could not be assigned to any coding- or non-coding RNA class, $20-23 \%$ of the reads mapped to repetitive sequences of the genome. Further $12-14 \%$ of the reads represented fragments of tRNAs. miRNAs made up 9$16 \%$ of the mapped reads (Fig. 1b). The sequence read length profiles revealed two distinct peaks around $22 \mathrm{nt}$ and $32 / 33 \mathrm{nt}$. As expected, we found that miRNAs represent the main fraction of reads within the size range of $20 \mathrm{nt}$ to $24 \mathrm{nt}$. However, most sRNAs across all libraries fell in the size range of $30 \mathrm{nt}$ to $34 \mathrm{nt}$, including the majority of tRFs, most of which derive from the $5^{\prime}$ end of mature tRNAs. In addition and even considerably exceeding the number of tRFs, sRNAs derived from intergenic regions of the genome make up the large proportion of 30-34 nt sized sRNAs (Fig. 1c, Supplementary Table S4).

Based on previously published results we assumed that this fraction represented piRNAs and we checked for typical piRNA characteristics $[45,46]$. First, we noted a clear bias $(77-78 \%)$ for uridine at the $5^{\prime}$ end $(1 \mathrm{U})$ which is typical for primary piRNAs and distinguished this

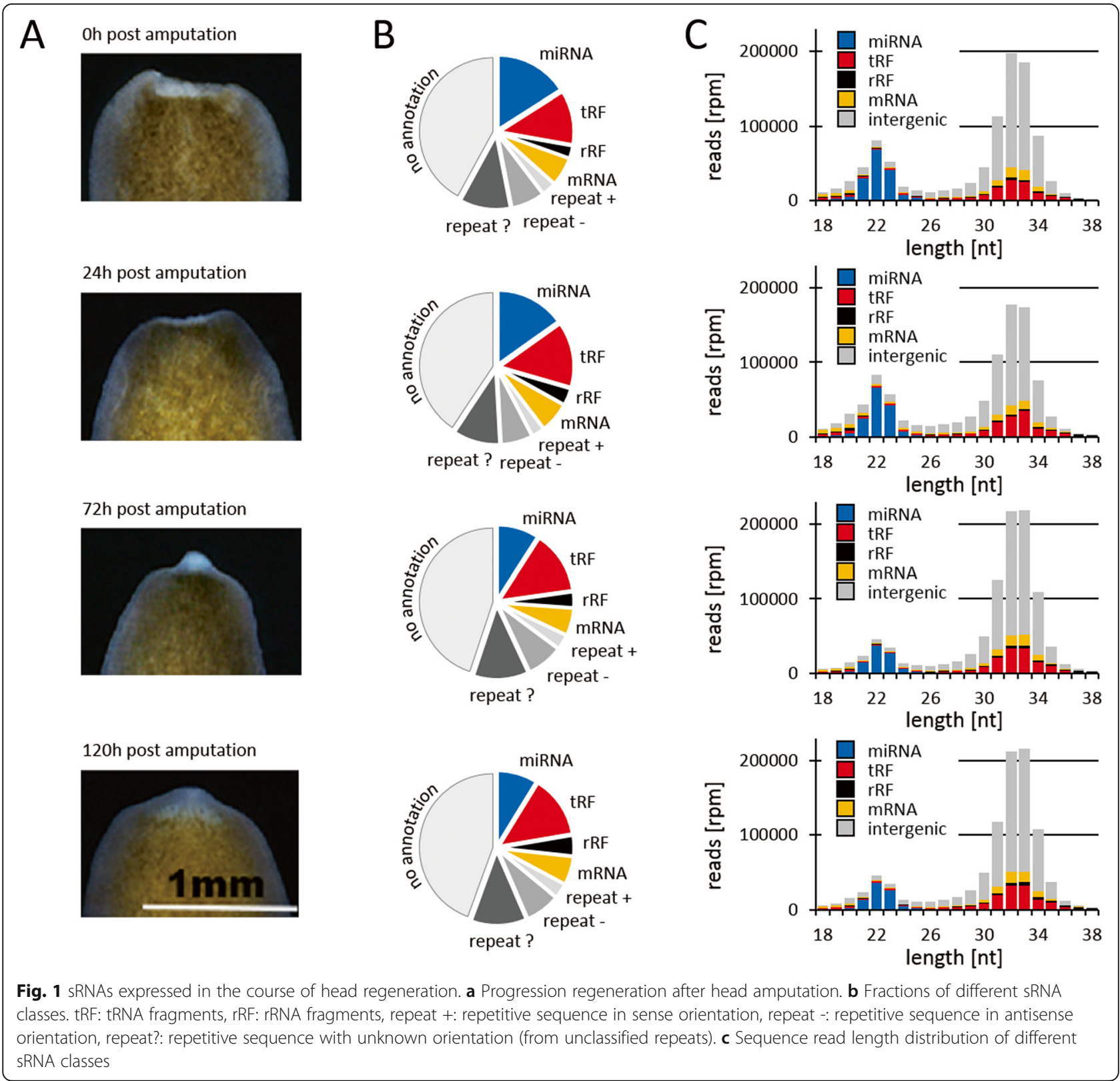


population from the other annotated sRNAs in D. japonica (Fig. 2a, Supplementary Table S5). In addition, considering the sub-fraction of repeat derived sequence reads, we observed a clear bias for sequences antisense to repeats (2.4-2.5-fold) suggesting a role in transposon silencing (Supplementary Table S6). Next we analyzed those reads that mapped to complementary strands of the genome and found a significant enrichment for $10 \mathrm{nt}$ $5^{\prime}$ overlaps (ping-pong signature), implicating the presence of primary and secondary piRNAs (ping-pong piRNAs, Fig. 2b, Supplementary Table S7). To verify that the observed ping-pong signature is generated by $30-34$ nt sRNAs, we checked the size of sequence reads that form ping-pong pairs and indeed found that the majority of ping-pong pairs combines sequence reads with a length between $30 \mathrm{nt}$ and $34 \mathrm{nt}$ (Fig. 2c, Supplementary Table S8). Finally, we used proTRAC to identify genomic piRNA clusters which in total yielded 283 distinct genomic loci that, while making up only $0.16 \%$ of the $D$. japonica genome, comprise on average $5 \%$ of the putative piRNAs which is very similar to findings regarding piRNA clustering in S. mediterranea (Friedländer et al. 2009, Supplementary Table S9). Together these results strongly support our assumption that the fraction of intergenic 30-35 nt sequence reads represents genuine piRNAs and we will bona fide refer to these sRNAs as piRNAs in the following. Noteworthy, given the fact that most predicted clusters are less than $10 \mathrm{~kb}$ in size with

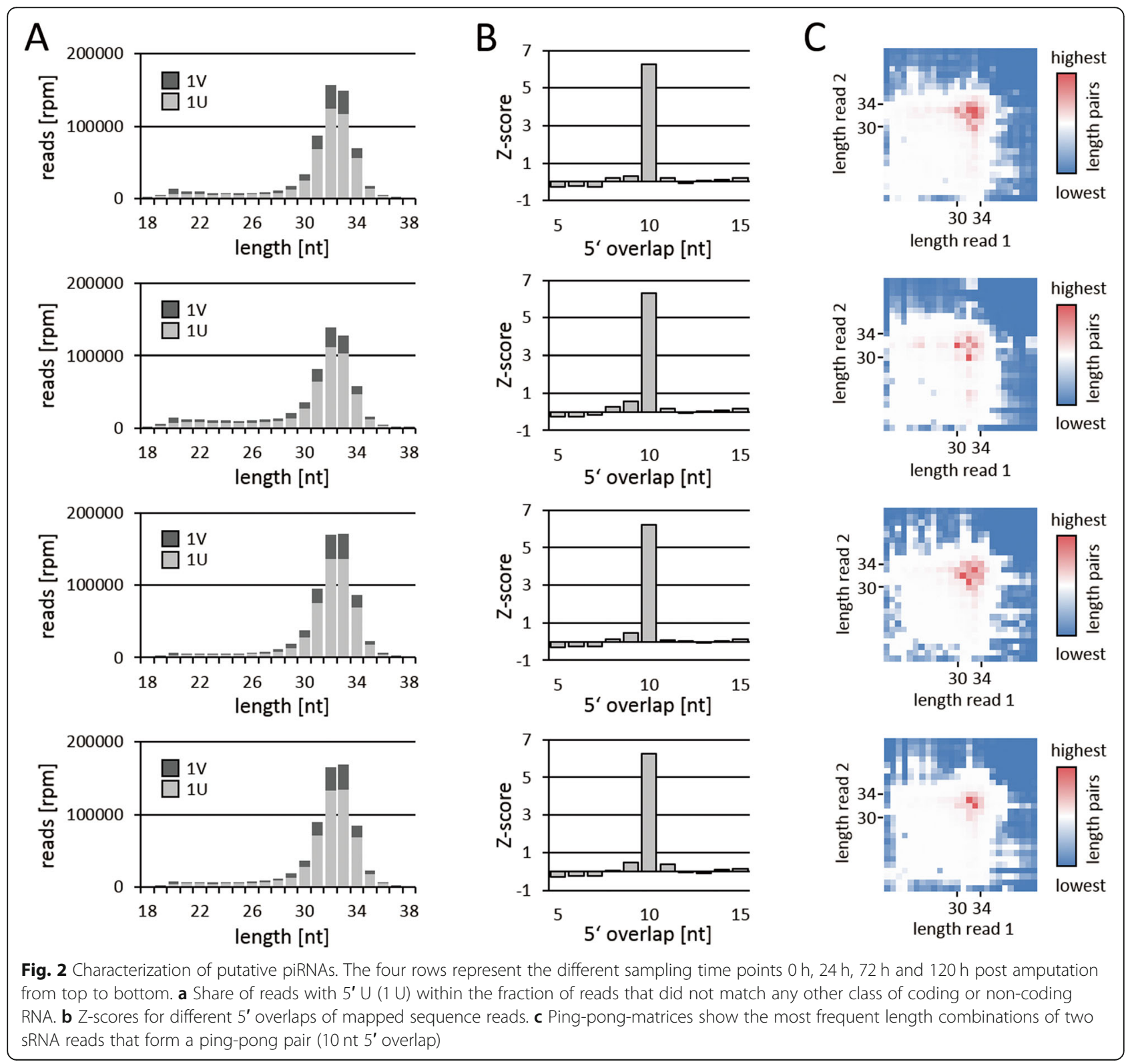


the largest cluster reaching $20 \mathrm{~kb}$, we cannot rule out the possibility that many of the predicted piRNA clusters in fact represent dispersed piRNA producing transposon copies. Therefor we will use the term piRNA cluster in sense of a piRNA producing locus in the following.

\section{Dynamic sRNA expression patterns during head regeneration}

We found that head amputation induced a global shift regarding the relative abundance of miRNA, tRFs and clustered piRNAs (Fig. 3a). While the relative abundance of miRNAs substantially decreases from $16.0 \%$ at the time of amputation to $8.8 \% 120 \mathrm{~h}$ post amputation, the abundance of tRFs increases moderately from $11.8 \%$ to $13.6 \%$. At the same time, although the overall fraction of putative piRNAs remains constant, the fraction of piRNAs arising from piRNA clusters drops from $8.9 \%$ to $2.9 \%$ (Fig. 3a). Since miRNAs, tRFs and piRNAs arise from different and largely independent pathways, we wanted to know whether the changes in their abundance are due to general effects regarding the particular biogenesis pathway, or alternatively are caused by more complex alterations in the composition of each small RNA pool, possibly representing a directed response to head amputation. In the first case we would expect all sRNAs of a particular class to show roughly the same degree of up- or down-regulation, while in the latter case each individual sequence would exhibit its individual expression course. In favor of the latter alternative, the expression profile for each sRNA class reveals that not
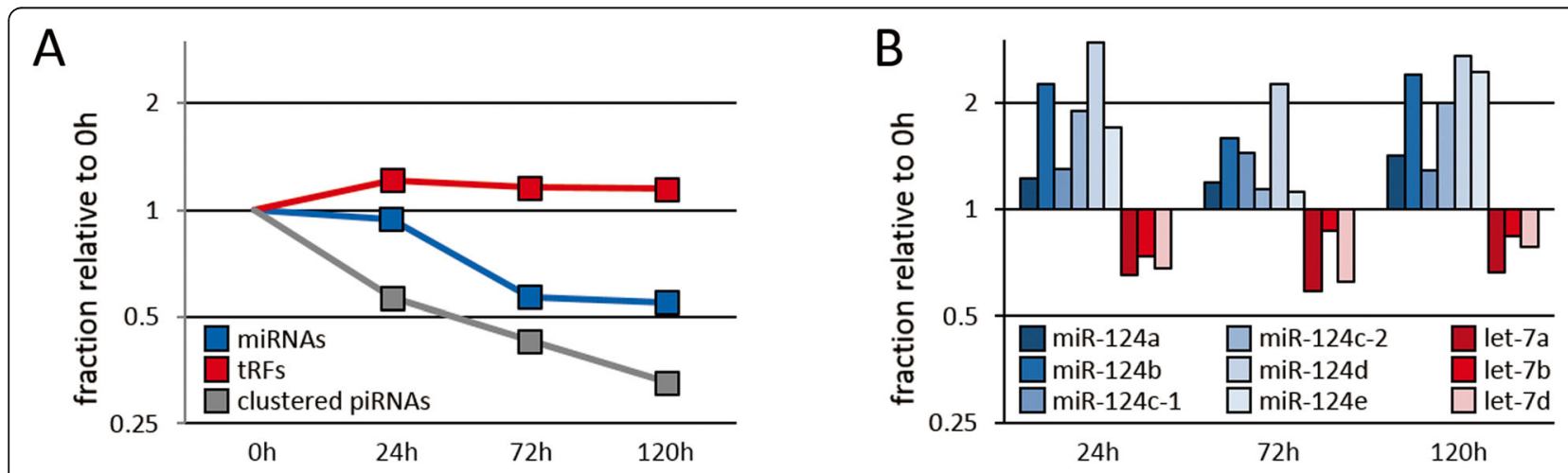

C

miRNAs
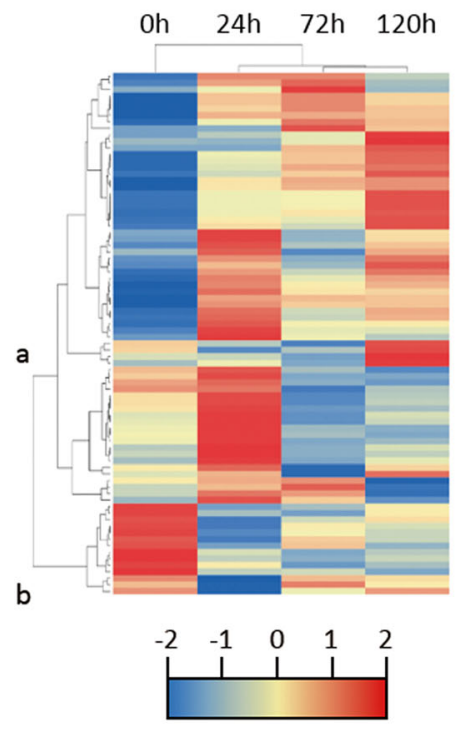

row Z-score

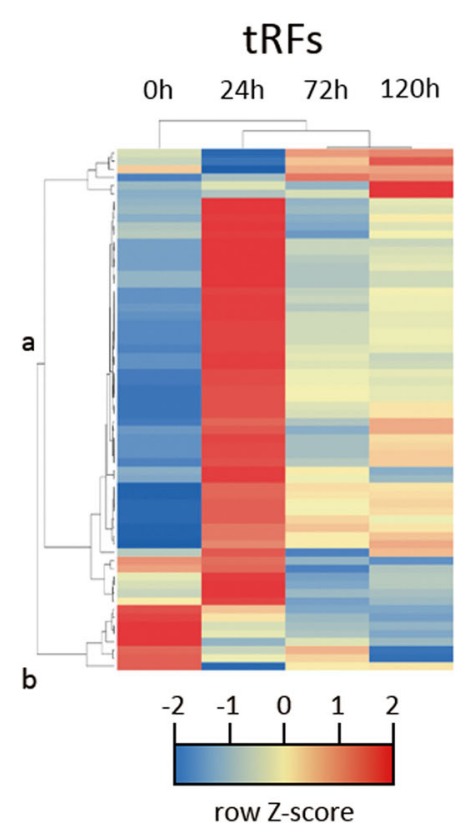

Fig. 3 Dynamic expression of sRNAs during head regeneration. a Expression changes of different sRNA classes in the course of regeneration. $\mathbf{b}$ Upregulation of mir-124 family and downregulation of let-7 miRNAs in regenerating animals. columns in heatmaps represent different sampling time points. Rows represent miRNA genes, source tRNAs and piRNA cluster loci, respectively 
only the overall abundance is subject to changes during head regeneration, but also the respective sequence composition (Supplementary Table S10 and S11). Regarding miRNAs, we observed a consistent upregulation of miR-124 family members following head amputation, while let-7a, let7b and let7d become less abundant (Fig. 3b). Similarly, different tRFs and piRNA clusters show contrary changes regarding their relative expression. Noteworthily, the hierarchical clustering pattern for the different sampling time points $(0 \mathrm{~h}, 24 \mathrm{~h}, 72$ $\mathrm{h}$ and $120 \mathrm{~h}$ post amputation) reveals that the global miRNA-, tRF- and piRNA cluster expression profile in regenerating animals $(24 \mathrm{~h}, 72 \mathrm{~h}$ and $120 \mathrm{~h}$ post amputation) is more similar to each other compared to that observed in animals directly after amputation ( 0 h, Fig. 3c). In each case we can distinguish two groups of sRNAs based on hierarchical clustering which we will refer to as group-a and group-b. While group-a sRNAs predominantly show an increased expression in regenerating animals, particularly in the early phase of regeneration $24 \mathrm{~h}$ post amputation, group-b sRNAs are less abundant following head amputation. As we would expect small RNAs that are involved in the process of head regeneration to be upregulated in regenerating animals, we assumed group-a sRNAs to be critical for regeneration. To gain support for this assumption, we performed antisense oligo-DNA mediated knockdown experiments focusing on selected sRNAs.

\section{Knockdown of different sRNAs induces severe regeneration defects}

To check whether the observed upregulation of specific small RNAs following head amputation is either a symptomatic consequence, or alternatively orchestrates the process of head regeneration, we performed knockdown experiments for selected small RNAs. Strikingly, while control animals transfected with scrambled oligomeric DNA regenerated normal heads and photoreceptors (PR) throughout, the vast majority of animals treated with $400 \mu \mathrm{M}$ anti-sRNA oligomeric DNA showed various types of PR defects, including the complete absence of PRs and cyclopia. In addition, even when the animals regenerated two PRs, these were often small and/or exhibited merely the light capturing pigment cells while lacking the white region around the pigment cells. A smaller number of animals showed lesions in the head region and subsequently lysed (Fig. 4a).

Whenever animals regenerated two PRs, we measured PR size (surface area) and the PR distance to each other, relative to the head diameter. For each knockdown condition, we found that PRs were significantly smaller $(p<$ 0.0001 ) and reached only $52-66 \%$ of the average surface area of control animals (Fig. 4b). Regarding the PR distance to each other we did not notice a significant shift of the mean distance compared to control animals, but, however, found that the variance was increased which frequently resulted in PRs with an exceptionally high or low distance to each other.

To check if regeneration-associated genes are potentially targeted by those eight miRNAs whose knock down resulted in impaired regeneration, we predicted target sites on the entirety of $D$. japonica mRNAs annotated with maker [47] using miranda [44]. We then compared the number of target sites on regenerationassociated genes (foxD, wnt1, beta-catenin-1, APC, NOTUM, CHD4, coe, pitx, patched) with the number of total mRNA target sites. We repeated this procedure with a control set including the eight most abundant miRNAs (bantam-a, miR-13, miR-17b, let-7a, miR-1c, lin-4, miR-281, let-7b) that did not show enrichment after head amputation. However, although we identified 33 putative target sites of phenotype-associated miRNAs on regeneration-associated genes, we found no evidence that these miRNAs target regeneration-associated genes more frequently than other genes, compared to the control set of miRNAs.

\section{Discussion}

Owing to their outstanding regenerative capabilities, planarians represent an important model organism to study molecular pathways connected to the process of regeneration. However, to assess whether findings from the widely used model $S$. mediterranea are likely applicable to other animals or not is difficult, since we often lack information on the evolutionary conservation of molecular pathways. As a first attempt to close this gap and to extend the currently available data $[48,49]$, we analyzed the changes in sRNA expression in response to head amputation in D. japonica. We show that while the miRNA fraction as a whole shrinks after head amputation, the expression of specific miRNAs increases. Knockdown of these miRNAs induces severe impairments of regeneration, demonstrating the functional importance of these miRNAs for the process of head regeneration.

Recently, it has been shown that the miR-124 family is crucial for regeneration of the brain and visual system in the planarian S. mediterranea. Remarkably, knockdown of Dugesia orthologs dja-miR-124c1 and dja-miR-124c2 resulted in similar phenotypes including head lesions, absence of eyes and cyclopia, suggesting a deeply conserved role of this miRNA in the process of regeneration. Interestingly, miR-124 is also highly expressed in human brain tissues where it functions as a master regulator of neurogenesis [50]. Further, experiments in Parkinson's Disease mouse models revealed that injection of miR-124 alleviated neurodegeneration and promoted neurogenesis [50, 51]. Given the deeply conserved role of miR-124 in regeneration and neurogenesis across distantly related species like planarians and humans, a 


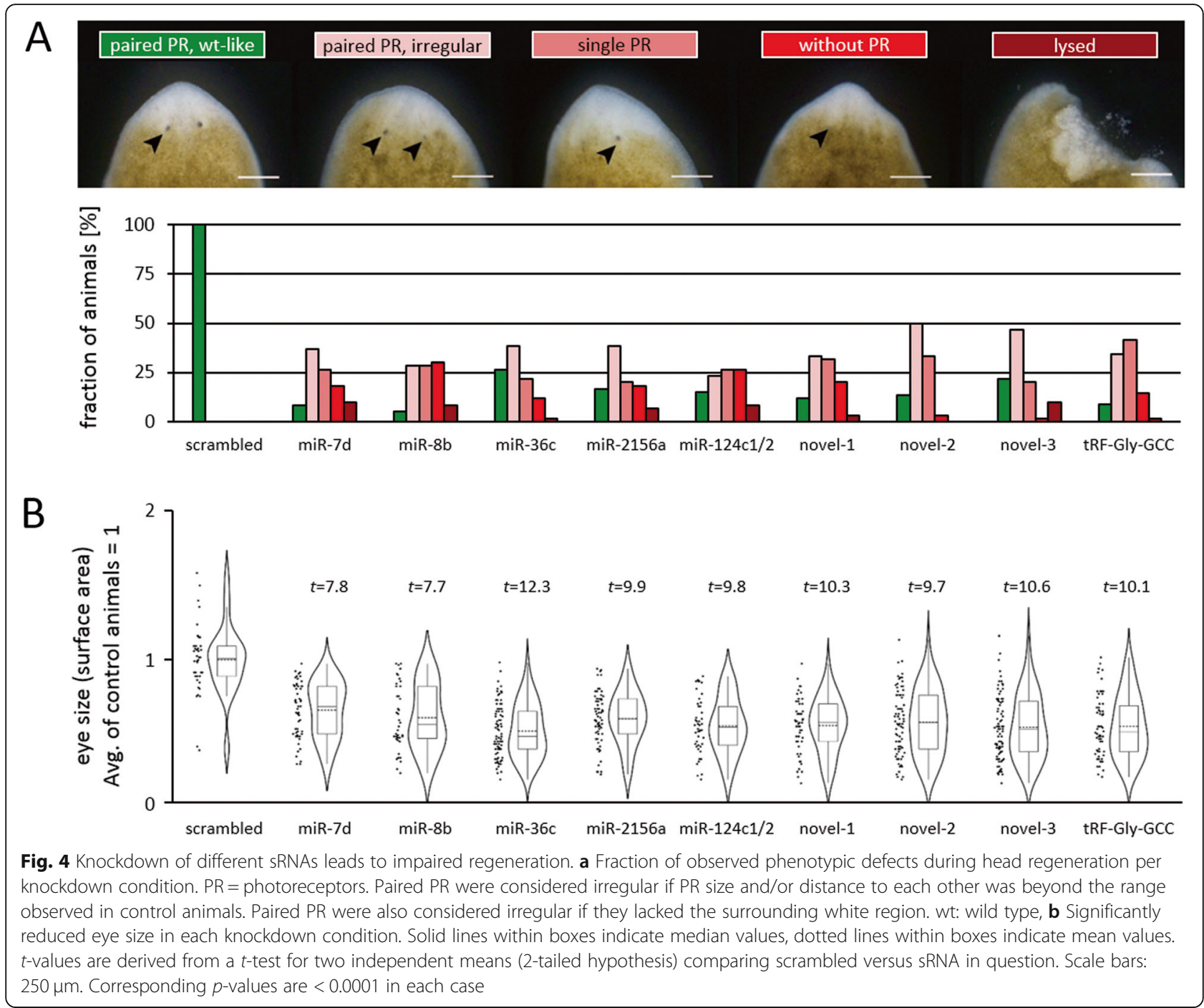

systematic functional examination of other small RNAs involved in planarian head regeneration appears promising, all the more, as regenerative therapies that base on treatment with miRNA mimics show encouraging results in the mouse model $[52,53]$.

Surprisingly, all of our knockdown experiments yielded very similar results regarding both the quality and quantity of phenotypes. Since these sRNAs lack obvious $5^{\prime}$ homology and thus likely target different sets of genes, we presume that they act critically in very early stages of regeneration, where any kind of dysregulation results in similar final defects.

Noteworthily, we show that sRNAs that are critical for planarian head regeneration not only include miRNAs, but also small RNAs with yet widely unknown functional potential such as tRFs. A number of recent studies has demonstrated that fragments of mature tRNAs can be more than pure degradation products, being involved in processes such as transposon regulation, global translational repression, sequence specific gene regulation, response to environmental stress and transgenerational epigenetics of metabolic disorders [54-63]. Interestingly, specific tRFs, including fragments of tRNA-Gly-GCC, have been linked to neurodevelopmental disorders by inducing a cellular stress response in mice [64]. By showing that $5^{\prime}$ tRF-Gly-GCC is upregulated in regenerating $D$. japonica animals, and that knockdown of $5^{\prime}$ tRF-Gly-GCC induces impaired head regeneration, we add yet another functional dimension to the biology of tRNA derived fragments.

Regarding a possible involvement of the piRNA pathway in regeneration, we found that the expression of piRNA clusters is greatly reduced upon head amputation. PIWI proteins represent markers for somatic stem cells in deep-branching metazoans $[65,66]$ and it was recently shown that a nuclear PIWI is required for cell differentiation in $D$. japonica by silencing transpsons [46]. We thus speculate that the downregulation of piRNA 
clusters reflects a shift from stem cell self-renewal in pre-amputation individuals, towards cell proliferation and differentiation in regenerating animals, possibly yielding a regeneration-specific population of piRNAs that is loaded onto PIWI proteins in regenerating animals. In fact, evidence for functional roles of the piRNA pathway in neurons of different invertebrate species exists [67].

\section{Conclusions}

In summary, our study confirms the deeply conserved role of miR-124 in the process of regeneration. It further reports a number of sRNAs, including 5' tRF-Gly-GCC, whose knockdown produces pursuant phenotypic defects, rendering these sRNAs attractive candidates for further functional investigations regarding their role during regeneration in different taxa including mammals.

\section{Methods}

\section{Housing conditions and regeneration experiments}

Head regeneration experiments were carried out with an asexual monophyletic strain of $D$. japonica which was originally obtained from Boshan, Shandong province, China. The animals were maintained at $21^{\circ} \mathrm{C}$ in mountain spring water and fed with beef liver paste once of week ad libitum. Animals were starved for 1 week prior to all experiments. Twenty animals were divided equally into four groups, each group include five animals. Each animal was decapitated just behind the auricle, and transferred into fresh water to regenerate. Different regeneration group animals were collected at $0 \mathrm{~h}, 24 \mathrm{~h}, 72$ $\mathrm{h}$, and $120 \mathrm{~h}$ after amputation, collected animals were homogenized in TRIzol (Invitrogen) immediately. Every five animals constitute a mixed pool of RNA library for RNA sequencing. All experiments involving animals in this study were approved by the Animal Welfare Committee of the Shandong University of Technology.

\section{RNA extraction, library preparation and sequencing}

Total RNA was extracted with TRIzol (Invitrogen) according to the manufacturer's instructions. Washed RNA was dissolved in $20 \mu \mathrm{l}$ nuclease-free water and the concentration was determined using the NANODROP 2000C system (Thermo scientific).

Illumina's TruSeq RNA Preparation Kit was used to purify 15-40 nt small RNAs from total RNA. Size selection and quality control were performed by BGI, Hong Kong. Libraries for each time point were sequenced on an Illumina HiSeq4000 sequencer (single-end, $50 \mathrm{bp}$ ). The raw reads were filtered according to the following criteria: 1) more than four bases had a quality score below 10 or more than six bases had a quality score below 13. 2) Reads containing homo-polymers, such as poly-A. 3) Reads with $5^{\prime}$ adapter contaminants or without 3' adapter sequence. 4) Reads without insert tag. 5) Reads with a length below $18 \mathrm{nt}$. Sequence data can be accessed at NCBI's Sequence Read Archive under the accession PRJNA549868.

\section{Genome annotation}

In order to obtain a comprehensive set of reference sequences allowing to classify our small RNAs, we annotated the genome of $D$. japonica [38] (assembly $\mathrm{Dj}$ Genome ver1.0) using tRNAscan for tRNA annotation (Supplementary Table S12), rnammer for rRNA annotation (Supplementary Table S13), maker for prediction of mRNA including UTRs (Supplementary Data S1), and RepeatMasker as well as WindowMasker for the annotation of repetitive DNA (Supplementary Table S14). tRNAscan v.1.3.1 and rnammer v.1.2 were run with default parameters. For mRNA annotation with the maker pipeline we used available nucleotide and peptide sequences from $S$. mediterranea and D. japonica obtained from NCBI nucleotide and protein database. We conducted RepeatMasker v.4.0.7 annotation using S. mediterranea and ancestral repeat sequences using the option -slow and the cross_match algorithm. Nonredundant repeats with a minimum size of $50 \mathrm{bp}$ annotated with WindowMasker v.1.0.0 were combined to a merged repeat annotation. For de novo prediction of miRNA genes we merged sRNA data from the different sampling time points and ran ShortStack [39] allowing 2 mismatches. The predicted miRNA precursor sequences were aligned to annotated miRBase stem-loop sequences with blastn and miRNA names were assigned according to the best given an E-value was below $10^{-6}$. Otherwise the miRNA gene was assumed to be novel.

\section{Small RNA mapping and annotation}

Clean sequence reads were mapped to the genome of $D$. japonica with SeqMap [40] allowing up to 3 mismatches. Subsequently we filtered the best hits for each sequence in terms of mismatch counts, allowing no more than one internal mismatch and two non-template 3 ' nucleotides using the custom Perl script SeqWrap.pl. The obtained map files were used as input for sRNA annotation with unitas [41] based on the obtained reference sequences (see above) using the options -slow -pp. The amount of small RNAs derived from repetitive DNA was calculated using the custom Perl script RMvsMAP.pl. piRNA clusters were predicted separately for each dataset with proTRAC v.2.4.2 with the option -pimax 35 to account for the presence of piRNAs $>32 \mathrm{nt}$. The predicted clusters were merged as described in Jehn et al. [42].

\section{Differential expression analysis}

Heatmaps for differential expression of miRNAs, tRNA fragments and piRNA clusters were created with 
heatmapper [43] applying clustering of both dimensions based on average linkage and using the Pearson distance measurement method. Colors refer to row Z-scores. Only miRNAs with at least 100 reads per million miRNAs, and tRFs with at least 100 reads per million tRFs were taken into account.

\section{GO term enrichment analysis}

To check if miRNAs that are upregulated following head amputation, thus possibly playing a role in the process of head regeneration, we selected miRNAs that were found to be at least 2-fold upregulated upon amputation $(n=5)$ while having a minimum average expression of 100 reads per million miRNA reads. For each miRNA we chose the most abundant isoform and predicted putative targets of these five miRNAs in combination as well as separately using miranda [44] with default settings, searching for target sites aligning to the 3' UTRs of predicted $D$. japonica mRNAs. We applied the same strategy to determine putative pathways regulated by the four novel $D$. japonica miRNAs. To assign GO terms to the de novo predicted mRNAs we used blastn to align predicted coding sequences of D. japonica to coding sequences of $S$. mediterranea for which GO term annotations are available [38]. We assumed coding sequences to be homologous if the alignments yielded E-values $\leq 10^{-6}$. We applied Chi-square tests with strict Bonferroni correction of alpha-error to identify GO terms that were enriched in the set of predicted target genes.

\section{Knockdown of selected small RNAs}

Based on de novo miRNA annotation and differential expression analysis of sequenced small RNAs, we selected a set of candidate small RNAs for knockdown experiments in regenerating animals (dja-mir-novel1/2/3, mir-124c-1/c-2, mir-2156a, mir-36c, mir-7d, mir-8b, and 5 '-tRF-Gly-GCC). Dugesia japonica planarians were retroauricular amputated. Head-less individuals were collected in 24-well plates (20-30 individuals per well, bottom area $1.9 \mathrm{~cm}^{2}$ ). Each antisense oligomeric DNA solution adjusted to the $5 \mathrm{uM}$ concentration was added to each well, collected animals were washed with approximately $100 \mathrm{ul}$ solution, then discard this solution and added 300ul new solution in each well (final concentration was $5 \mathrm{uM}$ ). The treated animals were cultured at $21^{\circ} \mathrm{C}$ for 7 days and the medium was changed by fresh antisense oligomeric DNA solution on the third day after decapitated. Phenotypes were recorded at 7 th day after amputation and each experiment was repeated three times. Paired photoreceptors of regenerating animals were considered irregular when i) the surface area of both photoreceptors differed by more than $33 \%$ and/or ii) the distance between both photoreceptors relative to the head diameter differed by more than $33 \%$ from to the scrambled control average.

\section{Supplementary Information}

The online version contains supplementary material available at https://doi. org/10.1186/s12864-020-07234-1.

Additional file 1: Supplemental_Data_S1. The reference sequences for mRNAs (including UTRs).

Additional file 2: Supplemental_Table_S1. The identified 36 miRNA genes with ShortStack.

Additional file 3: Supplemental_Table_S2. The enrichment of specific GO terms assigned to the putative targets of the novel miRNAs.

Additional file 4: Supplemental_Table_S3. The annotation results of small RNAs using the unitas annotation pipeline.

Additional file 5: Supplemental_Table_S4. The length distribution of small RNAs.

Additional file 6: Supplemental_Table_S5. Share of reads with 5' U $(1 \mathrm{U})$ within the fraction of reads that did not match any other class of coding or non-coding RNA.

Additional file 7: Supplemental Table S6. The sub-fraction of repeat derived sequence reads.

Additional file 8: Supplemental_Table_S7. The number of read pairs with 10 nt $5^{\prime}$ overlap.

Additional file 9: Supplemental_Table_S8. The size of sequence reads that form ping-pong pairs.

Additional file 10: Supplemental_Table_s9. The genomic piRNA clusters.

Additional file 11: Supplemental_Table_S10. The expression profile of miRNAs.

Additional file 12: Supplemental_Table_S11. The expression profile of tRFs.

Additional file 13: Supplemental_Table_S12. The reference sequences for tRNA.

Additional file 14: Supplemental_Table_S13. The reference sequences for rRNA.

Additional file 15: Supplemental_Table_S14. RepeatMasker as well as WindowMasker for the annotation of repetitive DNA

\section{Acknowledgements}

We would like to thank Chao Ning from Beijing Institute of genome research, Chinese Academy of Sciences and Huiguang Wu from Yangzhou University for technical comments on the study design.

Authors' contributions

$B Z, B L$ and ZC proposed the idea and designed the study. DR and ZC carried out the computational analysis and drafted the manuscript. SW, QP, XZ and $\mathrm{HL}$ performed the samples collection, RNA extraction and the knock down experiment. The authors read and approved the final version of the manuscript.

\section{Funding}

This research was supported by the National Natural Science Foundation of China (grants 31701308 to Z.C., 31572263 to B.Z.), Shandong Province Natural Science Foundation (grants ZR2014DM015 toX.Z).

Availability of data and materials

The small RNA-seq data generated in this study have been submitted to the NCBI BioProject database (http://www.ncbi.nlm.nih.gov/bioproject/) under accession number PRJNA549868.

Ethics approval and consent to participate

All experiments involving animals in this study were approved by the Animal Welfare Committee of Shandong University of Technology. 


\section{Consent for publication}

Not applicable.

\section{Competing interests}

The authors declare that they have no competing interests.

\section{Author details}

${ }^{1}$ School of Life Sciences, Shandong University of Technology, 266 Xincun Western Road, Zibo 255049, People's Republic of China. ${ }^{2}$ Institute of Organismic and Molecular Evolution (iOME), Anthropology,

Anselm-Franz-von-Bentzel-Weg 7, Johannes Gutenberg University, 55099 Mainz, Germany.

Received: 31 October 2019 Accepted: 17 November 2020

\section{Published online: 07 December 2020}

\section{References}

1. Reddien PW, Sanchez Alvarado A. Fundamentals of planarian regeneration Annu Rev Cell Dev Biol. 2004;20:725-57.

2. Forsthoefel DJ, Newmark PA. Emerging patterns in planarian regeneration. Curr Opin Genet Dev. 2009;19(4):412-20.

3. Salo E, Abril JF, Adell T, Cebria F, Eckelt K, Fernandez-Taboada E, et al. Planarian regeneration: achievements and future directions after 20 years of research. Int J Dev Biol. 2009;53(8-10):1317-27.

4. Brown DDR, Pearson BJ. A brain unfixed: unlimited neurogenesis and regeneration of the adult planarian nervous system. Front Neurosci. 2017;11:289.

5. Umesono Y, Watanabe K, Agata K. Distinct structural domains in the planarian brain defined by the expression of evolutionarily conserved homeobox genes. Dev Genes Evol. 1999;209(1):31-9.

6. Collins JJ 3rd, Hou X, Romanova EV, Lambrus BG, Miller CM, Saberi A, et al. Genome-wide analyses reveal a role for peptide hormones in planarian germline development. PLoS Biol. 2010;8(10):e1000509.

7. Lange CS, Steele VE. The mechanism of anterior-posterior polarity control in planarians. Differentiation. 1978;11(1):1-12.

8. Sarnat HB, Netsky MG. The brain of the planarian as the ancestor of the human brain. Can J Neurol Sci. 1985;12(4):296-302.

9. Agata K, Soejima Y, Kato K, Kobayashi C, Umesono Y, Watanabe K. Structure of the planarian central nervous system (CNS) revealed by neuronal cell markers. Zoolog Sci. 1998;15(3):433-40.

10. Sanchez Alvarado A. Planarian regeneration: its end is its beginning. Cell. 2006;124(2):241-5.

11. Mineta K, Nakazawa M, Cebria F, Ikeo K, Agata K, Gojobori T. Origin and evolutionary process of the CNS elucidated by comparative genomics analysis of planarian ESTs. Proc Natl Acad Sci U S A. 2003; 100(13):7666-71.

12. Owlarn S, Bartscherer K. Go ahead, grow a head! A planarian's guide to anterior regeneration. Regeneration (Oxf). 2016:3(3):139-55.

13. Fincher CT, Wurtzel O, de Hoog T, Kravarik KM, Reddien PW. Cell type transcriptome atlas for the planarian Schmidtea mediterranea. Science. 2018;360(6391):eaaq1736.

14. Witchley JN, Mayer M, Wagner DE, Owen JH, Reddien PW. Muscle cells provide instructions for planarian regeneration. Cell Rep. 2013;4(4):633-41.

15. Petersen CP, Reddien PW. A wound-induced Wnt expression program controls planarian regeneration polarity. Proc Natl Acad Sci U S A. 2009; 106(40):17061-6.

16. Petersen CP, Reddien PW. Polarized notum activation at wounds inhibits Wht function to promote planarian head regeneration. Science. 2011; 332(6031):852-5.

17. Gurley KA, Rink JC, Sanchez Alvarado A. Beta-catenin defines head versus tail identity during planarian regeneration and homeostasis. Science. 2008; 319(5861):323-7.

18. Iglesias M, Gomez-Skarmeta JL, Salo E, Adell T. Silencing of Smedbetacatenin 1 generates radial-like hypercephalized planarians. Development. 2008;135(7):1215-21.

19. Petersen CP, Reddien PW. Smed-betacatenin-1 is required for anteroposterior blastema polarity in planarian regeneration. Science. 2008; 319(5861):327-30

20. Rink JC, Gurley KA, Elliott SA, Sanchez Alvarado A. Planarian Hh signaling regulates regeneration polarity and links Hh pathway evolution to cilia. Science. 2009;326(5958):1406-10.
21. Yazawa S, Umesono Y, Hayashi T, Tarui H, Agata K. Planarian hedgehog/ patched establishes anterior-posterior polarity by regulating Wnt signaling Proc Natl Acad Sci U S A. 2009;106(52):22329-34.

22. Scimone ML, Lapan SW, Reddien PW. A forkhead transcription factor is wound-induced at the planarian midline and required for anterior pole regeneration. PLoS Genet. 2014;10(1):e1003999.

23. Vasquez-Doorman C, Petersen CP. Zic-1 expression in planarian neoblasts after injury controls anterior pole regeneration. PLoS Genet. 2014;10(7):e1004452.

24. Vogg MC, Owlarn S, Perez Rico YA, Xie J, Suzuki Y, Gentile L, et al. Stem celldependent formation of a functional anterior regeneration pole in planarians requires Zic and Forkhead transcription factors. Dev Biol. 2014; 390(2):136-48.

25. Pearson BJ, Sanchez Alvarado A. A planarian p53 homolog regulates proliferation and self-renewal in adult stem cell lineages. Development. 2010;137(2):213-21.

26. Scimone ML, Srivastava M, Bell GW, Reddien PW. A regulatory program for excretory system regeneration in planarians. Development. 2011;138(20):4387-98.

27. Zhu SJ, Hallows SE, Currie KW, Xu C, Pearson BJ. A mex3 homolog is required for differentiation during planarian stem cell lineage development. Elife. 2015:4:e07025.

28. Sen CK, Ghatak S. miRNA control of tissue repair and regeneration. Am J Pathol. 2015;185(10):2629-40.

29. Li N, Long B, Han W, Yuan S, Wang K. microRNAs: important regulators of stem cells. Stem Cell Res Ther. 2017;8(1):110

30. Fuller-Carter PI, Carter KW, Anderson D, Harvey AR, Giles KM, Rodger J. Integrated analyses of zebrafish miRNA and mRNA expression profiles identify miR-29b and miR-223 as potential regulators of optic nerve regeneration. BMC Genomics. 2015;16:591.

31. Ghibaudi M, Boido M, Vercelli A. Functional integration of complex miRNA networks in central and peripheral lesion and axonal regeneration. Prog Neurobiol. 2017;158:69-93.

32. van Battum EY, Verhagen MG, Vangoor VR, Fujita Y, Derijck A, O'Duibhir E, et al. An image-based miRNA screen identifies miRNA-135s as regulators of CNS axon growth and regeneration by targeting Kruppel-like factor 4. J Neurosci. 2018;38(3):613-30.

33. Fernandez-Ruiz I. Cardiac regeneration: a hydrogel-miRNA complex stimulates heart recovery. Nat Rev Cardiol. 2018;15(2):68-9.

34. Palakodeti D, Smielewska M, Lu YC, Yeo GW, Graveley BR. The PIWI proteins SMEDWI-2 and SMEDWI-3 are required for stem cell function and piRNA expression in planarians. RNA. 2008;14(6):1174-86.

35. Sasidharan V, Lu YC, Bansal D, Dasari P, Poduval D, Seshasayee A, et al. Identification of neoblast- and regeneration-specific miRNAs in the planarian Schmidtea mediterranea. RNA. 2013;19(10):1394-404.

36. Sasidharan V, Marepally S, Elliott SA, Baid S, Lakshmanan V, Nayyar N, et al. The miR-124 family of microRNAs is crucial for regeneration of the brain and visual system in the planarian Schmidtea mediterranea. Development. 2017:144(18):3211-23.

37. Lazaro EM, Harrath AH, Stocchino GA, Pala M, Baguna J, Riutort M. Schmidtea mediterranea phylogeography: an old species surviving on a few Mediterranean islands? BMC Evol Biol. 2011;11:274.

38. An Y, Kawaguchi A, Zhao C, Toyoda A, Sharifi-Zarchi A, Mousavi SA, et al. Draft genome of Dugesia japonica provides insights into conserved regulatory elements of the brain restriction gene nou-darake in planarians. Zoological Lett. 2018;4:24.

39. Axtell MJ. ShortStack: comprehensive annotation and quantification of small RNA genes. RNA. 2013;19(6):740-51.

40. Jiang $H$, Wong $W H$. SeqMap: mapping massive amount of oligonucleotides to the genome. Bioinformatics. 2008;24(20):2395-6.

41. Gebert D, Hewel C, Rosenkranz D. Unitas: the universal tool for annotation of small RNAs. BMC Genomics. 2017;18(1):644.

42. Jehn J, Gebert D, Pipilescu F, Stern S, Kiefer JST, Hewel C, et al. PIWI genes and piRNAs are ubiquitously expressed in mollusks and show patterns of lineage-specific adaptation. Commun Biol. 2018;1:137.

43. Babicki S, Arndt D, Marcu A, Liang Y, Grant JR, Maciejewski A, et al. Heatmapper: web-enabled heat mapping for all. Nucleic Acids Res. 2016; 44(W1):W147-53.

44. Enright AJ, John B, Gaul U, Tuschl T, Sander C, Marks DS. MicroRNA targets in drosophila. Genome Biol. 2003;5(1):R1.

45. Friedlander MR, Adamidi C, Han T, Lebedeva S, Isenbarger TA, Hirst M, et al. High-resolution profiling and discovery of planarian small RNAs. Proc Natl Acad Sci U S A. 2009;106(28):11546-51. 
46. Shibata N, Kashima M, Ishiko T, Nishimura O, Rouhana L, Misaki K, et al. Inheritance of a nuclear PIWI from pluripotent stem cells by somatic descendants ensures differentiation by silencing transposons in planarian. Dev Cell. 2016;37(3):226-37.

47. Cantarel BL, Korf I, Robb SM, Parra G, Ross E, Moore B, et al. MAKER: an easyto-use annotation pipeline designed for emerging model organism genomes. Genome Res. 2008;18(1):188-96.

48. Qin YF, Zhao JM, Bao ZX, Zhu ZY, Mai J, Huang YB, et al. Identification of small non-coding RNAs in the planarian Dugesia japonica via deep sequencing. Genomics. 2012;99(5):315-21.

49. Xu Z, Chen M, Ren Z, Zhang N, Xu H, Liu X, et al. Deep sequencing identifies regulated small RNAs in Dugesia japonica. Mol Biol Rep. 2013; 40(6):4075-81.

50. Saraiva C, Paiva J, Santos T, Ferreira L, Bernardino L. MicroRNA-124 loaded nanoparticles enhance brain repair in Parkinson's disease. J Control Release. 2016;235:291-305

51. Wang H, Ye Y, Zhu Z, Mo L, Lin C, Wang Q, et al. MiR-124 regulates apoptosis and autophagy process in MPTP model of Parkinson's disease by targeting to Bim. Brain Pathol. 2016;26(2):167-76.

52. Wang Y, Li Y, Zhang P, Baker ST, Wolfson MR, Weiser JN, et al. Regenerative therapy based on miRNA-302 mimics for enhancing host recovery from pneumonia caused by Streptococcus pneumoniae. Proc Natl Acad Sci U S A. 2019;116(17):8493-8.

53. Gao F, Kataoka M, Liu N, Liang T, Huang ZP, Gu F, et al. Therapeutic role of miR-19a/19b in cardiac regeneration and protection from myocardial infarction. Nat Commun. 2019;10(1):1802.

54. Yamasaki S, Ivanov P, Hu GF, Anderson P. Angiogenin cleaves tRNA and promotes stress-induced translational repression. J Cell Biol. 2009;185(1):35-42.

55. Gebetsberger J, Zywicki M, Kunzi A, Polacek N. tRNA-derived fragments target the ribosome and function as regulatory non-coding RNA in Haloferax volcanii. Archaea. 2012;2012:260909.

56. Sharma U, Conine CC, Shea JM, Boskovic A, Derr AG, Bing XY, et al. Biogenesis and function of tRNA fragments during sperm maturation and fertilization in mammals. Science. 2016;351(6271):391-6.

57. Chen Q, Yan M, Cao Z, Li X, Zhang Y, Shi J, et al. Sperm tsRNAs contribute to intergenerational inheritance of an acquired metabolic disorder. Science. 2016;351(6271):397-400.

58. Martinez G, Choudury SG, Slotkin RK. tRNA-derived small RNAs target transposable element transcripts. Nucleic Acids Res. 2017:45(9):5142-52.

59. Schorn AJ, Gutbrod MJ, LeBlanc C, Martienssen R. LTR-Retrotransposon control by tRNA-derived small RNAs. Cell. 2017;170(1):61-71 e11.

60. Gebetsberger J, Wyss L, Mleczko AM, Reuther J, Polacek N. A tRNA-derived fragment competes with mRNA for ribosome binding and regulates translation during stress. RNA Biol. 2017;14(10):1364-73.

61. Luo S, He F, Luo J, Dou S, Wang Y, Guo A, et al. Drosophila tsRNAs preferentially suppress general translation machinery via antisense pairing and participate in cellular starvation response. Nucleic Acids Res. 2018; 46(10):5250-68.

62. Liu S, Chen Y, Ren Y, Zhou J, Ren J, Lee I, et al. A tRNA-derived RNA fragment plays an important role in the mechanism of Arsenite -induced cellular responses. Sci Rep. 2018;8(1):16838.

63. Sarker G, Sun W, Rosenkranz D, Pelczar P, Opitz L, Efthymiou V, et al. Maternal overnutrition programs hedonic and metabolic phenotypes across generations through sperm tsRNAs. Proc Natl Acad Sci U S A. 2019;116(21): 10547-56.

64. Blanco S, Dietmann S, Flores JV, Hussain S, Kutter C, Humphreys P, et al. Aberrant methylation of tRNAs links cellular stress to neuro-developmental disorders. EMBO J. 2014;33(18):2020-39.

65. Juliano C, Wang J, Lin H. Uniting germline and stem cells: the function of Piwi proteins and the piRNA pathway in diverse organisms. Annu Rev Genet. 2011:45:447-69.

66. Ross RJ, Weiner MM, Lin H. PIWI proteins and PIWl-interacting RNAs in the soma. Nature. 2014;505(7483):353-9.

67. Rojas-Rios P, Simonelig M. piRNAs and PIWI proteins: regulators of gene expression in development and stem cells. Development. 2018;145(17): dev161786.

\section{Publisher's Note}

Springer Nature remains neutral with regard to jurisdictional claims in published maps and institutional affiliations.

Ready to submit your research? Choose BMC and benefit from:

- fast, convenient online submission

- thorough peer review by experienced researchers in your field

- rapid publication on acceptance

- support for research data, including large and complex data types

- gold Open Access which fosters wider collaboration and increased citations

- maximum visibility for your research: over $100 \mathrm{M}$ website views per year

At BMC, research is always in progress.

Learn more biomedcentral.com/submissions 